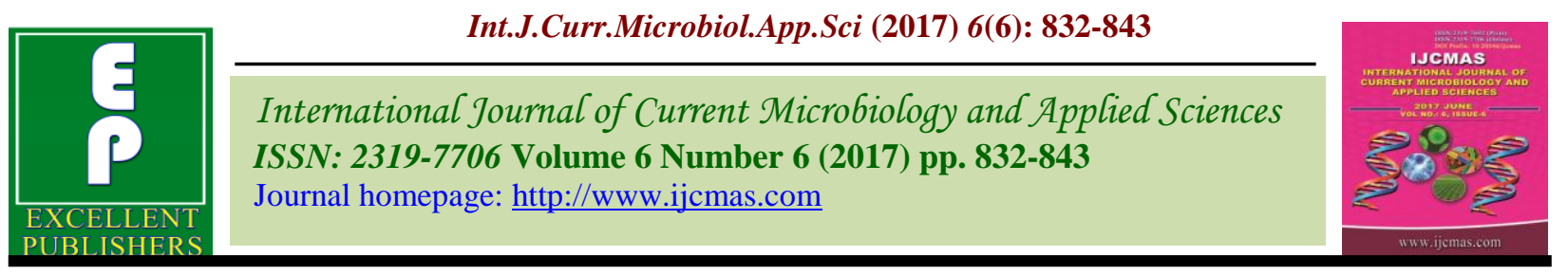

Original Research Article

https://doi.org/10.20546/ijcmas.2017.606.098

\title{
Genotypes against Major Diseases in Green Gram and Black Gram under Natural Field Conditions
}

\author{
A. Vijaya Bhaskar* \\ Aicrp Mullarp Subcentre, Regional Agricultural Research Station, Warangal, \\ PJTSAU, Telangana State, India \\ *Corresponding author
}

A B S T R A C T

\begin{tabular}{|c|}
\hline Keywords \\
\hline $\begin{array}{l}\text { Genotypes, } \\
\text { Leaf spot, } \\
\text { Powdery mildew, } \\
\text { Mung bean yellow } \\
\text { mosaic virus } \\
\text { Diseases in green } \\
\text { gram and black } \\
\text { gram. }\end{array}$ \\
\hline Article Info \\
\hline $\begin{array}{l}\text { Accepted: } \\
\text { 14 May } 2017 \\
\text { Available Online: } \\
\text { 10 June } 2017\end{array}$ \\
\hline
\end{tabular}

Green gram and Black gram genotypes/germplasms were evaluated to identify the sources of resistance to leaf spot, powdery Mildew and mung bean yellow mosaic virus (mymv) diseases. Screening was done under natural field conditions at Regional Agricultural Research Station (RARS), Warangal, Telangana State, India. The experimental material consisted fifty two AICRP + nine advanced Warangal Green gram entries with two checks and 10 state Warangal Black gram entries with a check, which were screened against the major diseases during Kharif-2015 at RARS, Warangal. Out of sixty three Green gram entries, only one KMP-13 was moderately resistant to Cercospora leaf spot disease, three entries viz., KMP-36, KMP-39 and KMP-41 were found highly resistant to powdery mildew, ten entries viz., KMP-13,19,20,22,23,24,40,45,MLGG-8 and WGG-42 were found immune to mung bean yellow mosaic virus disease. Out of eleven Black gram entries, only one MASH-338 was moderately resistant to Corynespora leaf spot disease, four entries viz., PU-31, MASH-338, LBG-752 and MBG-1050 were found moderately resistant to powdery mildew and two entries viz., PU-31 and MASH-338 were found immune to mung bean yellow mosaic virus disease. Resistants' can be exploited to develop high yielding varieties of Green gram and Black gram by breeding.

\section{Introduction}

Green gram and Black gram crops are major pulse crops of Telangana State. The less production of Green gram and Black gram is mainly attributed to low genetic yield potentiality, indeterminate growth habit, canopy architecture, low partitioning efficiency, cultivation in marginal land, biotic and abiotic stresses. Among biotic stresses, leaf spot, powdery mildew and mungbean yellow mosaic virus (mymv) are major diseases and have been found to appear in the epiphytotic form thereby causing immense loss in farmers' field of Telangana State.
Cercospora leaf spot was first known to be occurred in Delhi, India (Munjal et al., 1960) and is prevalent in all parts of the humid tropical areas of India, Bangladesh, Indonesia, Malaysia, Philippines, Taiwan as well as Thailand (Pandey et al., 2009). It becomes severe in the wet season causing $0.0 \%$ to 100.0 per cent yield loss (Quebral and Cagampang, 1970; Amin and Singh, 1987; Grewal, 1988; Iqbal et al., 1995; Pandey et al., 2009). Powdery mildew occurs across India and Southeast Asian countries and becomes severe in dry season causing $9.0 \%$ 
to 50.0 per cent yield loss (Reddy et al., 2008; Pandey et al., 2009). The powdery mildew occurs throughout the year under favorable conditions and it is more severe in late sown kharif crop.

A factor limiting breeding progress is that selection for powdery mildew resistance is confined to the cool-dry season on mungbean (Chankaew et al., 2013). Depending upon crop variety and location, disease incidence of MYMV was from $4 \%$ to $40 \%$ in Pakistan (Bashir et al., 2006). In several cases, leaves and other plant parts become completely yellow and the losses may be as high as $100 \%$ (Malik, 1991; Bashir et al., 2006). Singh et $a l .$, (2000) reported an incidence ranging from $0 \%$ to $58.5 \%$ among various varieties during their evaluation program for resistance against MYMV from Uttar Pradesh. MYMV disease leads to severe yield reduction not only in India, but also in Pakistan, Bangladesh and areas of South East Asia (Malathi et al., 2008 and Biswas et al., 2012) in Black gram.

Numerous attempts have been made for the identification of resistant sources against these diseases (Basandrai et al., 1999, 2003, 2011a; Iqbal et al., 2004; Raje and Rao, 2002; Reddy et al., 1994a, 2001 and 2008) of Green gram and Black gram.

Depending upon the temperature and humidity, these diseases spread rapidly in susceptible varieties. In Telangana state, it is cultivated in all most districts but prominently grown in Warangal, Khammam, Medak, Mahaboobunagar, Karimnagar, Nalgonda, Nizamabad and Rangareddy districts. Cultivation of resistant genotypes is an effective and cheaper method to combat the disease. Hence, several genotypes need to be screened to identify the source of resistance. Hence, an attempt was made to identify resistant genotypes against major diseases in Green gram and Black gram.

\section{Materials and Methods}

Trial was conducted in a Randomized Block Design (RBD) with two replications during Kharif-2015 at RARS, Warangal, Telangana state. Recommended agronomic practices were followed.

\section{Evaluation for leaf spot disease incidence on Green gram and Black gram}

Sixty three Green gram and eleven Black gram germplasms were evaluated under natural environmental field conditions at RARS, Warangal during Kharif-2015. Germplasms were planted in two rows of 4 meter length with row spacing of $40 \mathrm{~cm}$ and $10 \mathrm{~cm}$ between plants. The trial was laid out in $\mathrm{RBD}$ with two replications.

\section{Results and Discussion}

\section{Greengram}

\section{Leaf spot disease incidence}

Leaf spot disease incidence in sixty three Green gram germplasms lines was from $0 \%$ to $96 \%$. Based on the mean disease incidence of both replications during Kharif-2015, KMP13 was found moderately resistant, eight entries viz., KMP -19, 22, 30, 34,39,40,41 and MLGG-8 were moderately susceptible and remaining entries were susceptible to leaf spot disease (Table 1).

Out of 696 germplasms screened against leaf spot disease, 15 lines viz., (ML5, 443, 453, 515, 610, 611, 613, 682, 688, 713, 728, $735,746,759$ and 769) were found resistant to leaf spot disease (Singh et al., 2004). Jameel Akhtar et al., (2014) reported four genotypes viz., AKM 9910, IPM 02-5, ML 1299 and SML 668 were resistant to Cercospora leaf spot disease in Green gram. 


\section{Powdery mildew disease incidence}

Powdery mildew disease incidence in sixty three Greengram germplasms lines was from $0 \%$ to $90 \%$. Based on the mean disease incidence of both replications during Kharif2015, three entries viz., KMP-36,39 and 41 were found highly resistant, fourteen entries viz., KMP -2,3,5,19,20,24,30,34,38,42,47,52 and MLGG-8 were found resistant and remaining entries were susceptible to powdery mildew disease (Table 1). Out of 374 accessions, six entries of Green gram were found to be highly resistant viz., BL 849, BL 865, LM1668, PBM, PMB 63 and AKM 8803 (Divya Ramakrishnan et al., 2014). Similarly Jameel Akhtar et al., (2014) found 13 genotypes viz., KGS 83, MH 96-1, Pusa 572, GS 33-5, AKM 99-4, GS 21-5, COGG 936, ML 1299, TMB 47, HUM 1, MH 429, MH 429 and MH 530 were highly resistant reaction to powdery mildew disease in Green gram.

Several sources of resistance to Powdery mildew disease in mungbean have been reported (Hartman et al., 1993 and Reddy et al., 1994b). Genetic studies using different resistance sources revealed different modes of inheritance (Reddy et al., 1994a; Reddy, 2009; Sorajjapinun et al., 2005 and Kasettranan et al., 2009), suggesting that there are different mechanisms or genes conferring resistance to powdery mildew disease.

\section{Mung bean yellow mosaic virus disease incidence}

Yellow mosaic virus disease incidence in sixty three Green gram germplasms lines was from $0 \%$ to $100 \%$. Based on the mean disease incidence of both replications during Kharif2015, eleven entries viz., KMP$13,19,20,22,23,24,26,34,52$, MLGG-8 and WGG-42 were found immune, five entries viz., KMP-35, MGG-360, 373, 385 and MGG 395 were resistant and remaining entries were susceptible to mung bean yellow mosaic virus disease, out of sixty three entries (Table 2).

Out of 64 Mung bean lines, only six entries viz., AZRI-1, NCM-15-11, NCM-21, NCM11-8, 14063, AZRI-06 were found resistant to yellow mosaic virus disease in Green gram (Muhammad Hanif Munawar et al., 2014). Pathak and Jhamaria (2004) evaluated fourteen Mung bean varieties for resistance against YMV and found ML-5 and MUM-2 with resistance of $2.22 \%$ and $3.12 \%$ infection as against $100 \%$ infection in K-851a Check cultivar. Two entries viz., GG41 and GG42 were found resistant and GG52 showed moderate resistance to MYMV in Green gram (Peerajade et al., 2004).

\section{Black gram}

\section{Leaf spot disease incidence}

Leaf spot disease incidence in eleven Black gram germplasms lines was from $6 \%$ to $50 \%$. Based on the mean disease incidence of both replications during Kharif-2015, Only one entry MASH -338 was moderately resistant to leaf spot, six entries viz., PU-31, LBG-752, MBG-1042, MBG-1050, MBG-207 and WBG-26 were moderately susceptible and remaining entries were susceptible to leaf spot disease (Table 3). 
Based on the disease incidence, genotypes are categorized for their reaction to leaf spot disease as detailed, according to Mayee and Datar (1986) on Greengram and Blackgram

\begin{tabular}{|c|c|c|c|}
\hline $\begin{array}{l}\text { Disease } \\
\text { Scale }\end{array}$ & $\begin{array}{l}\text { Per cent } \\
\text { Leaf area } \\
\text { coverage }\end{array}$ & Description & Reaction \\
\hline 0 & 0 & No Symptom on the leaf & Immune (I) \\
\hline 1 & $<1$ & $\begin{array}{l}\text { Lesions small, pin head sized, covering less } \\
\text { than } 1 \% \text { leaf area }\end{array}$ & Resistant (R) \\
\hline 3 & $1-10$ & $\begin{array}{l}\text { Lesions } 1-2 \mathrm{~mm} \text { in diameter, covering } 1-10 \% \\
\text { of the leaf area }\end{array}$ & $\begin{array}{l}\text { Moderately } \\
\text { Resistant(MR) }\end{array}$ \\
\hline 5 & $11-25$ & $\begin{array}{l}\text { Lesions enlarged but not coalescing covering } \\
11-25 \% \text { of the leaf area }\end{array}$ & $\begin{array}{l}\text { Moderately } \\
\text { Susceptible (MS) }\end{array}$ \\
\hline 7 & $26-50$ & $\begin{array}{l}\text { Lesions coalescing covering } 26-50 \% \text { of leaf } \\
\text { area. }\end{array}$ & Susceptible (S) \\
\hline 9 & $>50$ & $\begin{array}{l}\text { Above } 50 \% \text { leaf area covered by large } \\
\text { coalescing lesions defoliation of leaves. }\end{array}$ & $\begin{array}{l}\text { Highly } \\
\text { Susceptible (HS) }\end{array}$ \\
\hline
\end{tabular}

Powdery mildew on Green gram and Black gram (0-5 Scale -Gawande and Patil, 2003)

\begin{tabular}{|l|l|l|l|}
\hline $\begin{array}{l}\text { Disease } \\
\text { Scale }\end{array}$ & $\begin{array}{l}\text { Per cent } \\
\text { Leaf } \\
\text { coverage }\end{array}$ & Description & Reaction \\
\hline 0 & 0 & Plants free from infection & $\begin{array}{l}\text { Highly Resistant } \\
\text { (HR) }\end{array}$ \\
\hline 1 & $1-10$ & $\begin{array}{l}\text { Plant showing traces up to 10 \% infection on } \\
\text { leaves, stem free from infection }\end{array}$ & Resistant (R) \\
\hline 2 & $10.1-25$ & $\begin{array}{l}\text { Slight infection with thin coating of powdery } \\
\text { growth on leaves covering 10.1-25 per cent } \\
\text { leaf area, slight infection on stem,pods usually } \\
\text { free }\end{array}$ & $\begin{array}{l}\text { Moderately } \\
\text { Resistant (MR) }\end{array}$ \\
\hline 3 & $25.1-50$ & $\begin{array}{l}\text { Dense powdery coating covering covering } \\
\text { 25.1 to 50\% leaf area,moderate infection on } \\
\text { stems,slight infection on pods }\end{array}$ & $\begin{array}{l}\text { Moderately } \\
\text { Susceptible (MS) }\end{array}$ \\
\hline 4 & $50.1-75$ & $\begin{array}{l}\text { Dense powdery coating covering 50.1 to 75\% } \\
\text { leaf area, stem heavily and pods moderately } \\
\text { infected.Infected portion turns greyish }\end{array}$ & Susceptible (S) \\
\hline 5 & $>75$ & $\begin{array}{l}\text { Severe infection with dense powdery growth, } \\
\text { covering more than 75 \% area of the whole } \\
\text { plant including pods, plants resulting in } \\
\text { premature defoliation and drying }\end{array}$ & $\begin{array}{l}\text { Highly Susceptible } \\
\text { (HS) }\end{array}$ \\
\hline
\end{tabular}


MungbeanYellow MosaicVirus on Green gram and Black gram (0-9 scale-Mayee and Datar, 1986)

\begin{tabular}{|l|l|l|l|}
\hline $\begin{array}{l}\text { Disease } \\
\text { Scale }\end{array}$ & $\begin{array}{l}\text { Per cent } \\
\text { leaf } \\
\text { coverage }\end{array}$ & Description & Reaction \\
\hline 0 & 0 & No visible Symptoms on leaves & Immune (I) \\
\hline 1 & $<1$ & Small yellow specks covering 0.1 to 1\% & Resistant (R) \\
\hline 3 & $1-10$ & $\begin{array}{l}\text { Yellow mottling of leaves covering 1.1 to } \\
10 \% \text { leaf area }\end{array}$ & $\begin{array}{l}\text { Moderately } \\
\text { Resistant (MR) }\end{array}$ \\
\hline 5 & $11-25$ & $\begin{array}{l}\text { Yellow mottling of leaves covering leaf area } \\
11 \text { to 25\% }\end{array}$ & $\begin{array}{l}\text { Moderately } \\
\text { Susceptible (MS) }\end{array}$ \\
\hline 7 & $26-50$ & $\begin{array}{l}\text { Yellow mottling and discoloration of 26 -50\% } \\
\text { leaf area }\end{array}$ & Susceptible (S) \\
\hline 9 & $>50$ & $\begin{array}{l}\text { Pronounced yellow mottling, discoloration of } \\
\text { leaves and pods, reduction in leaf size and } \\
\text { pod size, stunting of plants and no pod } \\
\text { formation (Above 50\% leaf area and pod) }\end{array}$ & $\begin{array}{l}\text { Highly Susceptible } \\
\text { (HS) }\end{array}$ \\
\hline
\end{tabular}

Table.1 Screening of Green gram genotypes against Leaf spot and Powdery Mildew Diseases

\begin{tabular}{|l|l|c|c|c|c|c|c|}
\hline S.No. & Genotypes & $\begin{array}{c}\text { Per cent } \\
\text { Leaf } \\
\text { area } \\
\text { infection }\end{array}$ & $\begin{array}{c}\text { Leaf } \\
\text { spot (0- } \\
\mathbf{9} \text { scale) }\end{array}$ & Reaction & $\begin{array}{c}\text { Per cent } \\
\text { Leaf } \\
\text { area } \\
\text { infection }\end{array}$ & $\begin{array}{c}\text { Powdery } \\
\text { Mildew } \\
\text { (0-5 scale) }\end{array}$ & Reaction \\
\hline 1 & KMP-1 & 30 & 7 & S & 12 & 2 & MR \\
\hline 2 & KMP-2 & 36 & 7 & S & 6 & 1 & R \\
\hline 3 & KMP-3 & 80 & 9 & HS & 8 & 1 & R \\
\hline 4 & KMP-4 & 42 & 7 & S & 60 & 4 & S \\
\hline 5 & KMP-5 & 40 & 7 & S & 4 & 1 & R \\
\hline 6 & KMP-6 & 38 & 7 & S & 56 & 4 & S \\
\hline 7 & KMP-7 & 35 & 7 & S & 20 & 2 & MR \\
\hline 8 & KMP-9 & 75 & 9 & HS & 60 & 4 & S \\
\hline 9 & KMP-11 & 82 & 9 & HS & 16 & 2 & MR \\
\hline 10 & KMP-12 & 86 & 9 & HS & 14 & 2 & MR \\
\hline 11 & KMP-13 & 5 & 3 & MR & 19 & 2 & MR \\
\hline 12 & KMP-14 & 70 & 9 & HS & 23 & 2 & MR \\
\hline 13 & KMP-17 & 78 & 9 & HS & 90 & 5 & HS \\
\hline 14 & KMP-18 & 84 & 9 & HS & 20 & 2 & MR \\
\hline 15 & KMP-19 & 22 & 5 & MS & 12 & 1 & R \\
\hline 16 & KMP-20 & 56 & 9 & HS & 14 & 1 & R \\
\hline 17 & KMP-22 & 20 & 5 & MS & 22 & 2 & MR \\
\hline 18 & KMP-23 & 90 & 9 & HS & 70 & 4 & S \\
\hline 19 & KMP-24 & 41 & 7 & S & 8 & 1 & R \\
\hline 20 & KMP-25 & 82 & 9 & HS & 68 & 4 & S \\
\hline
\end{tabular}




\begin{tabular}{|c|c|c|c|c|c|c|c|}
\hline 21 & KMP-26 & 80 & 9 & HS & 46 & 3 & MS \\
\hline 22 & KMP-28 & 41 & 7 & $\mathrm{~S}$ & 62 & 4 & $\mathrm{~S}$ \\
\hline 23 & KMP-30 & 23 & 5 & MS & 8 & 1 & $\mathrm{R}$ \\
\hline 24 & KMP-32 & 40 & 7 & $\mathrm{~S}$ & 65 & 4 & $S$ \\
\hline 25 & KMP-33 & 62 & 9 & HS & 70 & 4 & $\mathrm{~S}$ \\
\hline 26 & KMP-34 & 21 & 5 & MS & 4 & 1 & $\mathrm{R}$ \\
\hline 27 & KMP-35 & 92 & 9 & HS & 70 & 4 & $\mathrm{~S}$ \\
\hline 28 & KMP-36 & 100 & 9 & HS & 0 & 0 & HR \\
\hline 29 & KMP-38 & 86 & 9 & $\mathrm{HS}$ & 5 & 1 & $\mathrm{R}$ \\
\hline 30 & KMP-39 & 22 & 5 & MS & 0 & 0 & HR \\
\hline 31 & KMP-40 & 19 & 5 & MS & 32 & 3 & MS \\
\hline 32 & KMP-41 & 16 & 5 & $\mathrm{MS}$ & 0 & 0 & $\mathrm{HR}$ \\
\hline 33 & KMP-42 & 96 & 9 & $\mathrm{HS}$ & 8 & 1 & $\mathrm{R}$ \\
\hline 34 & KMP-44 & 42 & 7 & $S$ & 44 & 3 & MS \\
\hline 35 & KMP-45 & 60 & 9 & HS & 7 & 1 & $\mathrm{R}$ \\
\hline 36 & KMP-46 & 32 & 7 & $\mathrm{~S}$ & 68 & 4 & $\mathrm{~S}$ \\
\hline 37 & KMP-47 & 83 & 9 & HS & 6 & 1 & $\mathrm{R}$ \\
\hline 38 & KMP-48 & 46 & 7 & $S$ & 70 & 4 & $\mathrm{~S}$ \\
\hline 39 & KMP-52 & 40 & 7 & $\mathrm{~S}$ & 3 & 1 & $\mathrm{R}$ \\
\hline 40 & MGG-313 & 81 & 9 & $\mathrm{HS}$ & 32 & 3 & MS \\
\hline 41 & MGG-339 & 76 & 9 & $\mathrm{HS}$ & 40 & 3 & MS \\
\hline 42 & MGG-359 & 70 & 9 & HS & 82 & 5 & HS \\
\hline 43 & MGG-360 & 62 & 9 & HS & 14 & 2 & $\mathrm{MR}$ \\
\hline 44 & MGG-370 & 58 & 9 & HS & 16 & 2 & MR \\
\hline 45 & MGG-373 & 82 & 9 & HS & 60 & 4 & $\mathrm{~S}$ \\
\hline 46 & MGG-385 & 74 & 9 & HS & 18 & 2 & MR \\
\hline 47 & MGG-386 & 86 & 9 & $\mathrm{HS}$ & 41 & 3 & $\mathrm{MS}$ \\
\hline 48 & MGG-387 & 90 & 9 & $\mathrm{HS}$ & 80 & 5 & HS \\
\hline 49 & MGG-395 & 92 & 9 & HS & 15 & 2 & MR \\
\hline 50 & MLGG-1 & 66 & 9 & HS & 40 & 3 & $\mathrm{MS}$ \\
\hline 51 & MLGG-2 & 72 & 9 & HS & 66 & 4 & $\mathrm{~S}$ \\
\hline 52 & MLGG-3 & 54 & 9 & HS & 42 & 3 & $\mathrm{MS}$ \\
\hline 53 & MLGG-4 & 76 & 9 & HS & 20 & 2 & MR \\
\hline 54 & MLGG-5 & 80 & 9 & HS & 18 & 2 & MR \\
\hline 55 & MLGG-6 & 70 & 9 & $\mathrm{HS}$ & 70 & 4 & $\mathrm{~S}$ \\
\hline 56 & MLGG-7 & 42 & 7 & $S$ & 44 & 3 & MS \\
\hline 57 & MLGG-8 & 21 & 5 & $\mathrm{MS}$ & 8 & 1 & $\mathrm{R}$ \\
\hline 58 & MLGG-9 & 84 & 9 & HS & 70 & 4 & $\mathrm{~S}$ \\
\hline 59 & PANT-M2 & 68 & 9 & $\mathrm{HS}$ & 42 & 3 & MS \\
\hline 60 & PANT-M3 & 75 & 9 & HS & 36 & 3 & MS \\
\hline 61 & PBM-1 & 88 & 9 & HS & 20 & 2 & MR \\
\hline 62 & WGG-37 S.check & 38 & 7 & $\mathrm{~S}$ & 80 & 5 & HS \\
\hline 63 & WGG-42 S.check & 40 & 7 & $\mathrm{~S}$ & 20 & 2 & MR \\
\hline
\end{tabular}

[Resistant(R), Susceptible(S), Moderately Resistant (MR), Moderately Susceptible (MS), Highly Susceptible (HS)] 
Table.2 Screening of Green gram genotypes against Mung bean

Yellow Mosaic Virus (MYMV) disease

\begin{tabular}{|c|c|c|c|c|}
\hline S.No. & Genotypes & $\begin{array}{c}\text { Per cent Leaf area } \\
\text { infection }\end{array}$ & $\begin{array}{c}\text { MYMV } \\
\text { (0-9 scale) }\end{array}$ & Reaction \\
\hline 1 & KMP-1 & 40 & 7 & $\mathrm{~S}$ \\
\hline 2 & KMP-2 & 22 & 5 & MS \\
\hline 3 & KMP-3 & 24 & 5 & MS \\
\hline 4 & KMP-4 & 75 & 9 & HS \\
\hline 5 & KMP-5 & 88 & 9 & HS \\
\hline 6 & KMP-6 & 20 & 5 & MS \\
\hline 7 & KMP-7 & 46 & 7 & $S$ \\
\hline 8 & KMP-9 & 92 & 9 & HS \\
\hline 9 & KMP-11 & 19 & 5 & MS \\
\hline 10 & KMP-12 & 96 & 9 & HS \\
\hline 11 & KMP-13 & 0 & 0 & I \\
\hline 12 & KMP-14 & 20 & 5 & MS \\
\hline 13 & KMP-17 & 18 & 5 & MS \\
\hline 14 & KMP-18 & 46 & 7 & $\mathrm{~S}$ \\
\hline 15 & KMP-19 & 0 & 0 & I \\
\hline 16 & KMP-20 & & 0 & I \\
\hline 17 & KMP-22 & 0 & 0 & I \\
\hline 18 & KMP-23 & 0 & 0 & I \\
\hline 19 & KMP-24 & 0 & 0 & I \\
\hline 20 & KMP-25 & 100 & 9 & HS \\
\hline 21 & KMP-26 & 0 & 0 & I \\
\hline 22 & KMP-28 & 90 & 9 & HS \\
\hline 23 & KMP-30 & 46 & 7 & $\mathrm{~S}$ \\
\hline 24 & KMP-32 & 23 & 5 & MS \\
\hline 25 & KMP-33 & 19 & 5 & MS \\
\hline 26 & KMP-34 & 0 & 0 & I \\
\hline 27 & KMP-35 & 0.6 & 1 & $\mathrm{R}$ \\
\hline 28 & KMP-36 & 46 & 7 & $\mathrm{~S}$ \\
\hline 29 & KMP-38 & 98 & 9 & HS \\
\hline 30 & KMP-39 & 86 & 9 & HS \\
\hline 31 & KMP-40 & 7 & 3 & MR \\
\hline 32 & KMP-41 & 76 & 9 & HS \\
\hline 33 & KMP-42 & 40 & 7 & $S$ \\
\hline 34 & KMP-44 & 92 & 9 & HS \\
\hline 35 & KMP-45 & 8 & 3 & MR \\
\hline 36 & KMP-46 & 96 & 9 & HS \\
\hline 37 & KMP-47 & 46 & 7 & $\mathrm{~S}$ \\
\hline 38 & KMP-48 & 80 & 9 & HS \\
\hline 39 & KMP-52 & 0 & 0 & I \\
\hline 40 & MGG-313 & 75 & 9 & HS \\
\hline
\end{tabular}




\begin{tabular}{|c|c|c|c|c|}
\hline 41 & MGG-339 & 8 & 3 & MR \\
\hline 42 & MGG-359 & 4 & 3 & MR \\
\hline 43 & MGG-360 & 0.5 & 1 & $\mathrm{R}$ \\
\hline 44 & MGG-370 & 22 & 5 & MS \\
\hline 45 & MGG-373 & 6 & 3 & $\mathrm{R}$ \\
\hline 46 & MGG-385 & 4 & 3 & $\mathrm{R}$ \\
\hline 47 & MGG-386 & 20 & 5 & MS \\
\hline 48 & MGG-387 & 23 & 5 & MS \\
\hline 49 & MGG-395 & 6 & 3 & $\mathrm{R}$ \\
\hline 50 & MLGG-1 & 28 & 7 & $\mathrm{~S}$ \\
\hline 51 & MLGG-2 & 13 & 5 & MS \\
\hline 52 & MLGG-3 & 15 & 5 & MS \\
\hline 53 & MLGG-4 & 17 & 5 & MS \\
\hline 54 & MLGG-5 & 78 & 9 & $\mathrm{HS}$ \\
\hline 55 & MLGG-6 & 44 & 7 & $\mathrm{~S}$ \\
\hline 56 & MLGG-7 & 82 & 9 & HS \\
\hline 57 & MLGG-8 & 0 & 0 & I \\
\hline 58 & MLGG-9 & 18 & 5 & MS \\
\hline 59 & PANT-M2 & 72 & 9 & $\mathrm{HS}$ \\
\hline 60 & PANT-M3 & 79 & 9 & $\mathrm{HS}$ \\
\hline 61 & PBM-1 & 88 & 9 & $\mathrm{HS}$ \\
\hline 62 & WGG-37 S.check & 82 & 9 & $\mathrm{HS}$ \\
\hline 63 & WGG-42 R.check & 0 & 0 & I \\
\hline
\end{tabular}

[Immune (I), Resistant(R), Moderately Resistant (MR), Susceptible(S), Moderately Susceptible (MS), Highly susceptible (HS)]

Table.3 Screening of Black gram genotypes against Leaf spot and Powdery Mildew diseases

\begin{tabular}{|l|l|c|c|c|c|c|c|}
\hline $\begin{array}{l}\text { S. } \\
\text { No. }\end{array}$ & $\begin{array}{c}\text { Genotypes } \\
\text { Per cent } \\
\text { Leaf } \\
\text { area } \\
\text { infection }\end{array}$ & $\begin{array}{c}\text { Leaf } \\
\text { Spot } \\
\text { Scale } \\
\mathbf{( 0 - 9 )}\end{array}$ & Reaction & $\begin{array}{c}\text { Per cent } \\
\text { Leaf } \\
\text { area } \\
\text { infection }\end{array}$ & $\begin{array}{c}\text { Powdery } \\
\text { Mildew } \\
\text { scale } \\
\mathbf{( 0 - 5} \\
\text { scale) }\end{array}$ & Reaction \\
\hline 1 & PU-31 & 18 & 5 & MS & 23 & 2 & MR \\
\hline 2 & MASH-338 & 6 & 3 & MR & 15 & 2 & MR \\
\hline 3 & LBG-752 & 22 & 5 & MS & 21 & 2 & MR \\
\hline 4 & MBG-1044 & 44 & 7 & S & 40 & 3 & MS \\
\hline 5 & MBG-1045 & 35 & 7 & S & 32 & 3 & MS \\
\hline 6 & MBG-1050 & 20 & 5 & MS & 11 & 2 & MR \\
\hline 7 & MBG-1051 & 50 & 7 & S & 60 & 4 & S \\
\hline 8 & WBG-26 & 23 & 5 & MS & 38 & 3 & MS \\
\hline 9 & MBG-207 & 20 & 5 & MS & 30 & 3 & MS \\
\hline 10 & MBG-1047 & 46 & 7 & S & 28 & 3 & MS \\
\hline 11 & MBG-1042 & 25 & 5 & MS & 50 & 3 & MS \\
\hline
\end{tabular}

[Resistant(R), Moderately Resistant (MR), Susceptible(S), Moderately Susceptible (MS)] 
Table.4 Screening of Black gram genotypes against Yellow Mosaic Virus disease

\begin{tabular}{|l|l|c|c|c|}
\hline $\begin{array}{l}\text { S. } \\
\text { No. }\end{array}$ & Genotypes & $\begin{array}{c}\text { Per cent Leaf } \\
\text { area infection }\end{array}$ & $\begin{array}{c}\text { YMV scale } \\
(\mathbf{0 - 9})\end{array}$ & Reaction \\
\hline 1 & PU-31 & 0 & 0 & $\mathrm{I}$ \\
\hline 2 & MASH-338 & 0 & 0 & $\mathrm{I}$ \\
\hline 3 & LBG-752 & 0.8 & 1 & $\mathrm{R}$ \\
\hline 4 & MBG-1044 & 9 & 3 & MR \\
\hline 5 & MBG-1045 & 6 & 3 & MR \\
\hline 6 & MBG-1050 & 15 & 5 & MS \\
\hline 7 & MBG-1051 & 0.6 & 1 & $\mathrm{R}$ \\
\hline 8 & WBG-26 & 30 & 7 & S \\
\hline 9 & MBG-207 & 42 & 7 & S \\
\hline 10 & MBG-1047 & 7 & 3 & MR \\
\hline 11 & MBG-1042 & 50 & 7 & S \\
\hline
\end{tabular}

[Immune (I), Resistant(R), Moderately Resistant (MR), Susceptible(S), Moderately Susceptible (MS)]

Out of 14 genotypes of screened Black gram, one entry KUG 216 was found as highly resistant whereas three genotypes viz., BS 23, IPU 02-43 and B 3-8-8 were recorded as resistant to leaf spot disease (Jameel Akhtar et al., 2014).

\section{Powdery mildew disease incidence}

Powdery mildew disease incidence in eleven Black gram germplasms lines was from $11 \%$ to $60 \%$. Based on the mean disease incidence of both replications during Kharif-2015, only four entries i.e.PU-31, MASH -338, LBG-752 and MBG-1050 were found moderately resistant and remaining entries susceptible to powdery mildew disease (Table 3 ).

Out of 126 genotypes screened, none of them were found to be immune, however, three genotypes viz., LBG-17, LBG-685 and LBG$685 \times \mathrm{VT}$ (F2-F3) were found to be resistant to powdery mildew disease in Black gram (Channaveeresh et al., 2014). Jameel Akhtar et al., (2014) noticed five genotypes viz., Pant U 31, BS 2-3, IPU 02-43, KU 323 and KU 99-21 were highly resistant to powdery mildew disease.

\section{Yellow Mosaic Virus disease incidence}

Yellow mosaic virus disease incidence in eleven Black gram germplasms lines was from $0 \%$ to $50 \%$. Based on the mean disease incidence of both replications during Kharif2015, two entries viz.,PU-31 and MASH-338 were found immune, one entry LBG-752 was resistant and remaining entries were susceptible to yellow mosaic virus disease, out of 11 entries(Table 4).

Ganapathy et al., (2003) evaluated 71 urdbean genotypes to identify resistance against mungbean yellow mosaic virus, urdbean leaf crinkle virus and leaf curl virus and found five genotypes namely RU 2229,VBG 86,2KU 54,VBG 89 and SU16 were highly resistant to MYMV. Out of 45 genotypes screened, 19 lines (viz., PU-31, PU-205,PU 1075,IC-1704,IC-11668,IC-37978, IC-49203, MASH-1-1,IC -6110, PDBG-10, PU-30, MASH-114, PU-35,IC-59702,TBG-104,PU19,TU94-2,MASH338 and IC-14691) were free from disease with one score/resistant (Prasanthi et al., 2013). Out of 56 screened genotypes against YMV, 22 entries (viz., PU202,205,206,207,208,209,210,P-1051,P-1051, P-1052,P-1053,P-1058,P-1059,P-1060,P- 
1061,P-1062,P-1064, P-1065, P-1070, P1075,P-715 and PU-31) showed resistance to YMV (Obaiah et al., 2013).

\section{Multiple resistances}

Only one entry KMP-13 was found resistant to leaf spot and mung bean yellow mosaic virus diseases. Two entries (viz., KMP-52 and MLGG-8) were found resistant to powdery mildew and yellow mosaic virus diseases and remaining entries were susceptible to the above three diseases in Green gram.

Only one entry Mash-338 was found moderately resistant to leaf spot and powdery mildew diseases and LBG-752 entry was found resistant to powdery mildew and yellow mosaic virus diseases in Black gram.

\section{Acknowledgments}

The author are grateful to the Dr S Gupta, P.C, Mullarp, IIPR, Kanpur, for providing genotypes and Financial support was provided by PJTSA University officers for this study.

\section{References}

Amin, K.S. and Singh, R.A. 1987. Diseases of mung, urd and pea and their management. In Proceedings of National Seminar on Plant Protection in Field crops, CPPTI, Hyderabad, India, pp. 203-217.

Basandrai, A.K., Gartan, D., Basandrai, D. and Kalia, V. 1999. Blackgram (Phaseolus mungo) germplasm against different diseases. Indian J Agri. Sci., 69: 506-508.

Basandrai, D., Basandrai, A.K., Sharma, V., Devlash, R. and Gautam, N.K. 2011a. Evaluation for multiple resistances to yellow mosaic, anthracnose, Cercospora leaf spot and powdery mildew in urdbean germplasm. Plant Dis. Res., 26(2): 166.

Basandrai, D., Basandrai, A.K., Singh, I. and Kalia, V. 2003. Multiple disease resistance against anthracnose, Cercospora leaf spot, powdery mildew and mungbean yellow mosaic virus in blackgram (Vigna mungo). J. Mycol. Plant Pathol., 33: 56-58.

Bashir, M., A.R. Jamali, and Z. Ahmed. 2006. Genetic resistance in mungbean and mashbean germplasm against mungbean yellow mosaic begomovirus. Mycopath., 4(2): 1-4.

Biswas, K.K., Tarafdar, A., and Biswas, K. 2012. Viral diseases and its mixed infection in mungbean and urdbean: Major biotic constraints in production of food pulses in India. In Asha Sinha, B. K. Sharma and Manisha Srivastava (Eds.), Modern trends in microbial biodiversity of natural ecosystem. New Delhi: Biotech Books. pp. 301-317.

Chankaew, S., Somta, P., Isemura, T., Tomooka, N., Kaga, A., Vaughan, D.A. and Srinives, P. 2013. Quantitative trait locus mapping reveals conservation of major and minor loci for powdery mildew resistance in four sources of resistance in mungbean [Vigna radiata (L.) Wilczek]. Mol. Breed., 32: 121130.

Channaveeresh, T.S., Shripad kulkarni and Vijaykumar, G. 2014. Evaluation of blackgram genotypes for resistance to powdery mildew caused by Erysiphe polygoni DC. Karnataka J. Agric. Sci., 27(1): 85-87.

Divya Ramakrishnan, C.K. and Savithramma, D.L. 2014. Screening of mungbean germplasm for powdery mildew disease resistance. Int. J. Agron. Agri. Res., Vol. 4, No. 6, p. 16-21.

Ganapathy, T., Kuruppiah, R. and Gunasekaran, K. 2003. Identifying the source of resistance for mungbean 
yellow mosaic virus (MYMV), urd bean leaf crinkle virus and leaf curl virus disease in urdbean (Vigna mungo (L.) Hepper). In: Annual Meeting and Symposium on Recent Develop-ments in the Diagnosis and Management of Plant Diseases for Meeting Global Challenges, December 18-20, 2003, University of Agricultural Sciences, Dharwad, 30p.

Gawande, V.L., and Patil, J.V. 2003. Genetics of powdery mildew (Erysiphe polygoni DC.) resistance in mungbean (Vigna radiata (L.)Wilezck). Crop Protect, 22: 567-571.

Grewal, J.S. 1988. Disease of pulse crops An overview. Indian Phytopath., 41: 114.

Hartman, G.L., Wang, T.C. and Kim, D. 1993. Field evaluation of mungbeans for resistance to Cercospora leaf spot and powdery mildew. Int. J. Pest Manage., 39: 418-421.

Iqbal, S.M.A., Gafoor, A., Bashir, M. and Malik, B.A. 1995. Estimation of losses in yield Components of mungbean due to Cercospora leaf spot. Pak. J. Phytopathol., 7: 80-81.

Iqbal, S.M.A., Zubair, M. and Haqqani, A.M. 2004. Resistance in mungbean to Cercospora Leaf spot disease. Int. J. Agri. Biol., 6(5): 792-793.

Jameel Akhtar, H.C. Lal, Yogesh Kumar, P.K. Singh, Jyotirmoy Ghosh, Zakauallah Khan and N.K. Gautam. 2014. Multiple disease resistance in greengram and blackgram germplasm and management through chemicals under rain-fed conditions. Legume Res., 37(1): $101-109$.

Kasettranan, W., Somta, P. and Srinives, P. 2009. Genetics of the resistance to powdery mildew disease in mungbean (Vigna radiata (L.) Wilczek). J. Crop Sci. Biotechnol., 12(1): 37-42.
Malathi, V.G. and John, P. 2008. Mungbean yellow mosaic virus. In: Encyclo. Virol, Third edn., 8: 364-372.

Malik, I.A. 1991. Breeding for resis-tance to MYMV and its vector in Pakistan. In: Green, S.K. and Kim, D. (Eds.), Mungbean Yellow Mosaic Disease: Proceedings of an Inter-national Workshop. Bangkok, Thailand. July 23, 1991. AVRDC, Taiwan. 79 Pp.

Mayee, C.D. and Datar, V.V. 1986. Phytopathometry, Department of Plant pathology, Marathwada Agricultural University, Parbhani Technical Bull., No.1. 145-146.

Muhammad Hanif Munawwar, Asghar Ali and Shahid Riaz Malik. 2014. Identification of resistance in mungbean and mashbean germplasm against mungbean yellow mosaic virus. Pakistan J. Agric. Res., 27(2): 129-135.

Munjal, R.L., Lall, G. and Chona, B.L. 1960. Some Cercospora species from IndiaIV. Indian Phytopath., 13: 144-149.

Obaiah, S., Bhaskara Reddy, B.V., Eswara Reddy, N.P. and Siva Prasad, Y. 2013. Screening of some blackgram [Vigna mungo (L.) Hepper] genotypes for resistance to yellow mosaic virus. Curr. Biotica, 7(1\&2): 96-100.

Pandey, S., Sharma, M., Kumari, S., Gaur, P.M, Chen, W., Kaur, L., Macleod, W., Basandrai, A.K., Basandrai, D., Bakr, A., Sandhu, J.S., Tripathi, H.S. and Gowda, C.L.L. 2009. Integrated foliar diseases management of legumes. In: Grain Legumes: Genetic improvement, Management and Trade, Eds. By Masood Ali et al., pp.143-161. Indian Society of Pulses Research and Development, Indian Institute of Pulses Research, Kanpur, India.

Pathak, A.K. and S.L. Jhamaria. 2004. Evaluation of mungbean (Vigna radiata L.) varieties to yellow mosaic virus. $J$. Mycol. Plant Pathol., 34(1): 64-65. 
Prasanthi, L., Bhaskara Reddy, B.V., B., Geetha, Ramya Jyothi and Abhishek. 2013. Molecular marker for screening yellow mosaic disease resistance in blackgram [Vigna mungo (L.) Hepper]. Electronic J. Plant Breed., 4(2): 11371141

Peerajade, D.A., Ravikumar, R.L. and Rao M.S.L. 2004. Screening of local mungbean collections for powdery mildew and yellow mosaic virus resistance. Indian J. Pulses Res., 17(2): 190-191.

Quebral, F.C. and Cagampang, I.C. 1970. Influence of Cercospora leaf spot control on yield on mungbean. Agriculture Los Banos, 10: 7-12.

Raje, R.S. and Rao, S.K. 2002. Screening of mungbean (Vigna radiata L. Wilczek) germplasm for yellow mosaic virus and Cercospora leaf spot. Legume Res., 25: 99-19.

Reddy, K.S., Pawar, S.E., and Bhatia, C.R. 1994a. Inheritance of powdery mildew (Erysiphe polygoni D.C.) resistance in mungbean (Vigna radiata L. Wilczek). Theoretical and Appl. Genetics, Vol.88, 945-948.

Reddy, K.S., Pawar, S.E., Wanjari, K.B. and Bhatia, C.R. 1994b. Development of powdery mildews resistant and high yielding varieties of mungbean. In: International Symposium on Pulses Research, New Delhi, 146-147.

Reddy, K.S. 2009. Identification and inheritance of a new gene for powdery mildew resistance in mungbean (Vigna radiata L. Wilczek). Plant Breeding, 128: 521-523.

Reddy, K.S., Dhanasekar, P. and Dhole, V.J. 2008. A review on powdery mildew disease resistance in mungbean. J. Food Legumes, 21(3): 151-155.

Reddy, K.S., Pawar, S.E. and Bhatia, C.R. 2001. Host response of Vigna radiata genotypes to powdery mildew infection. Indian Phytopath., 54(1): 117-120.

Singh, B.R., Chandra, S. and Ram, S. 2000. Evaluation of mungbean varieties against yellow mosaic virus. Annals $\mathrm{Pl}$. Prot. Sci., 8(2): 233-280.

Singh Gurdip, Sharma, Y.R., Shanmugasundaram, S., Shih, S.L. and Green, S.K. 2004. Improving income and nutrition by incorporating mungbean in cereal fallows in the IndoGangetic Plains of South Asia DFID Mungbean Project for 2002-2004. Proceedings of the final workshop and planning meeting, Status of Mung bean yellow mosaic virus resistance breeding, Punjab Agricultural University, Ludhiana, Punjab, India, 2731 May 2004 pp. 204-213.

Sorajjapinun, W., Rewthongchum, S., Koizumi, M. and Srinives, P. 2005. Quantitative inheritance of resistance to powdery mildew disease in mungbean (Vigna radiata (L.) Wilczek). SABRAO J. Breed. Genetics, 37: 91-96.

\section{How to cite this article:}

Vijaya Bhaskar, A. 2017. Genotypes Against Major Diseases In Green Gram And Black Gram Under Natural Field Conditions. Int.J.Curr.Microbiol.App.Sci. 6(6): 832-843. doi: https://doi.org/10.20546/ijcmas.2017.606.098 